\title{
Retos contemporáneos a la ética en el proceso de la publicación científica
}

\author{
Andrés M. Pérez-Acosta*, Leonardo Amaya ${ }^{* *}$ \\ Universidad del Rosario
}

El reciente retiro formal del artículo "Análisis factorial exploratorio: bases conceptuales y metodológicas", publicado previamente en la Revista Argentina de Ciencias del Comportamiento (RACC) (RACC, 2017), nos motivó a proponer a la comunidad psicológica latinoamericana, por medio de esta nota editorial, una nueva aproximación sistemática a los retos contemporáneos a la ética en el proceso de la publicación científica, que cunden globalmente los campos de conocimiento.

En el ámbito académico internacional resuenan casos cada vez más extremos, como el de la investigadora italiana Carmine Finelli (Finelli et al., 2016), quien recibió el encargo de la revista Annals of Internal Medicine para evaluar un artículo y terminó robándolo, literalmente, al publicarlo como propio en otra revista (Dansinger, 2017); o el sorprendente caso de revisores falsos (en muchos casos eran los mismos autores que terminaban examinando su propio artículo, luego de dar la sugerencia de nombres reales de investigadores con un correo falso que solo manejaban los autores para autoevaluarse), que desembocó, el 20 de abril pasado, en una retractación masiva de 107 artículos publicados en la revista Tumor Biology (Springer, 2017).

Todo lo anterior ha convertido a la palabra "retractación" (en inglés: retraction) en la palabra de moda. No en vano, el blog Retraction
Watch $^{1}$ ya completa más de 14000 seguidores, incluyéndonos. Sin embargo, la retractación de artículos por parte de las revistas es el resultado común de un conjunto de conductas distintas, que requieren descripción, clasificación, explicación e intervención urgentes, sin llegar al extremo de proponer la condena a muerte a los investigadores imputados, como está sucediendo en China, país azotado por este tipo de faltas (El Espectador, 2017). Las acciones en cuestión están poniendo cada vez más en tela de juicio la credibilidad de la investigación científica. La genuina motivación de búsqueda de conocimiento, propia de los jóvenes investigadores, está siendo opacada por el enriquecimiento individual, el poder o el prestigio social (Gutiérrez, 2015).

Por supuesto, ya han corrido ríos de tinta sobre este tema (Benos et al., 2005; Hudson et al., 2006), incluyendo el contexto latinoamericano (López López, 2014; Gutiérrez, 2015). En ese sentido, nuestra pretensión es unirnos al esfuerzo de concienciación de nuestros antecesores y plantear algunos pequeños avances, siguiendo la estrategia de Benos et al. (2005) de tomar como eje el proceso de publicación científica (sometimiento, evaluación y publicación). Básicamente, lo que planteamos es ampliar la clasificación mencionada hacia el eje de la investigación científica: desde la elaboración del proyecto y su financiación hasta la indexación

* Correo electrónico: andres.perez@urosario.edu.co

**Correo electrónico: leonardo.amaya@urosario.edu.co

http://retractionwatch.com/ 
(visibilidad e impacto) de los artículos previamente publicados, a manera de sándwich de las etapas estrictamente relacionadas con la publicación.

Pero antes de un nuevo esfuerzo taxonómico, se requiere una propuesta conceptual, con base en dos preguntas básicas: (i) ¿qué es un reto a la ética de la publicación científica? y (ii) ¿quiénes están involucrados? En ese sentido, nos lanzamos a plantear nuestra respuesta sintética: un reto a la ética de la publicación científica puede definirse como una conducta corrupta, individual o colectiva, que desafía en términos morales o legales a cualquiera de los pasos estándar que deben seguir todos los actores involucrados en el proceso (autores, colaboradores, financiadores, editores, evaluadores e indexadores). En ese sentido, los autores no son las únicas personas que pueden quedar comprometidas en un reto a la ética de la publicación. Ya vimos cómo una persona bajo el rol de revisor se convirtió en autora fraudulenta del manuscrito sometido. Pero también un editor podría intentar modificar las citaciones de sus artículos aceptados, sin consultar a los autores, para aumentar artificialmente el factor de impacto o el índice $\mathrm{H}$ de su revista. $\mathrm{O}$ un funcionario de una agencia financiadora de investigación podría presionar por favores o propinas para dar su visto bueno. Ya no se requiere imaginación para anticipar este tipo conductas.

Con respecto a la taxonomía, los autores de esta editorial efectuamos una primera aproximación en la serie Experiencias Éticas (No. 4, abril 2017) del portal Ética Psicológica ${ }^{2}$, iniciativa de divulgación y formación de la Asociación Colombiana de Facultades de Psicología (Ascofapsi), el Colegio Colombiano de Psicólogos (Colpsic) y la Universidad del Rosario, Bogotá, Colombia. Abordamos los siguientes temas en formato de video-entrevista:

2 http://www.eticapsicologica.org a. Conflicto de interés (Ética Psicológica, 2017)

b. Plagio y autoplagio (Ética Psicológica, 2017a)

c. Manipulación de datos (Ética Psicológica, 2017b)

d. Sometimiento paralelo de artículos (Ética Psicológica, 2017c)

e. Retractación de artículos en revistas indexadas (Ética Psicológica, 2017d)

f. Reflexión final, educativa y crítica (Ética Psicológica, 2017e)

Ya en esta serie clasificamos los casos en tres momentos: (i) antes, (ii) durante y (iii) después del proceso de publicación científica. A continuación, mencionaremos algunos riesgos de cada momento:

1. Antes del proceso de publicación cientifica: conflicto de interés no declarado (Langer et al., 2012).

2. Durante del proceso de publicación cientifi$c a$ : autoría inmerecida o insuficiente (Kwok, 2005); plagio y autoplagio (Piña López, 2017); fabricación, manipulación o imposibilidad de replicación de los datos (Peterson et al., 2012); sometimiento paralelo.

3. Después del proceso de publicación cientifi$c a$ : alteraciones del contenido originalmente publicado (diferentes de una fe de erratas); manipulación de la indexación — visibilidad o impacto de la revista (Falagas \& Alexiou, 2008)-.

Como fue previamente mencionado, la consecuencia común creciente de todas estas conductas es la retractación de los artículos (en proceso de publicación o publicados) por parte de los editores $\mathrm{y}$, por supuesto, de los indexadores que ya hayan agregado el ítem en cuestión. Cuanta más visibilidad tenga la revista en bases de datos académicas y otras fuentes secundarias, el retiro del artículo comprometido es una secuencia quirúrgica más delicada y dolorosa. Por fortuna para los editores, el COPE (Comité Internacional de Ética en 
Publicaciones) ya dispone de una muy útil guía de retractación (COPE, 2009).

\section{Comentario final: explicación e intervención}

Uno de los casos más emblemáticos de conducta fraudulenta en publicación científica fue protagonizado por el psicólogo social holandés Diederik Stapel (Wikipedia, 2017). Este exprofesor de la Universidad de Tilburg fue suspendido por fabricar y manipular datos en sus reportes de investigación. Al menos 55 publicaciones estaban afectadas y, obviamente, fueron retractadas. Además de la gravedad del caso, nos interesa resaltarlo por dos razones: por tratarse de un investigador en nuestra disciplina (psicología) y por la explicación que él mismo ofreció de su conducta, en el reporte interno de la institución y en un libro posterior a su imputación (Stapel, 2014). Si bien, él nunca negó su gravísima falta ética y su responsabilidad individual, también afirmó que no soportó la presión de publicar cada vez más y en mejores revistas.

Justamente, el asunto de la presión por productividad fue también hallado en un par de estudios sobre salud laboral en profesores universitarios: uno en Holanda (Tijdink et al., 2013) y otro en Colombia (Gómez Ortiz, Perilla Toro \& Hermosa Rodríguez, 2015). El primero encontró indicios de niveles preocupantes de síndrome de burnout en profesores de medicina asociados con la presión excesiva. El segundo estudio llegó a la siguiente conclusión:

Tanto los profesores como las directivas universitarias necesitan ser más conscientes de los riesgos para la salud de los profesores, asociados al incremento de la presión de las instituciones para mejorar su competitividad y la calidad de sus servicios. Esta calidad se mide usualmente con indicadores tales como el número y tipo de publicaciones y otras que no necesariamente reflejan el bienestar o la salud de los miembros de la comunidad que produce esos resultados (pp. 198-199).

Ante la evidencia de esta situación, en dos momentos de la presente década, colectivos internacionales de investigadores científicos efectuaron dos importantes manifestaciones públicas: la Declaración de San Francisco (2012) y el Manifiesto de Leiden $(2015)^{4}$. En ambos documentos se llama la atención sobre la necesidad de una adecuada evaluación de los resultados de investigación, la cual no se debe sustentar de forma exclusiva en indicadores cuantitativos (por ejemplo, factor de impacto de las revistas o índice $\mathrm{H}$ de los autores). No se pretenden eliminar los indicadores cuantitativos, sino que estos se vean acompañados siempre con evaluaciones cualitativas proporcionadas por pares expertos para efectos de toma de decisiones en el contexto académico (por ejemplo, financiaciones, ascensos, contrataciones, despidos, premios, etc.).

Finalmente, se hace necesario complementar los esfuerzos de reformas en las políticas públicas y privadas de administración de la ciencia, con programas sistemáticos de educación temprana y continua en aspectos éticos en la investigación. La ignorancia sobre estos temas nunca será una excusa; en cambio sí puede ser un agravante (Benos et al., 2015).

\section{Referencias}

Benos, D. J., Fabres, J., Farmer, J., Gutierrez, J. P., Hennessy, K., Kosek, D., et al. (2005). Ethics and scientific publication. Advances in Physiology Education, 29(2), 59-74. DOI:10.1152/ advan.00056.2004

Dansinger, M. (enero, 2017). Dear Plagiarist: A Letter to a Peer Reviewer Who Stole and Published Our Manuscript as His Own. Annals of Internal Medicine. Recuperado de http://annals.org/aim/

\footnotetext{
http://www.ascb.org/dora/

$4 \quad$ http://www.ingenio.upv.es/es/manifiesto\#.WYAJqog1_IU
} 
article/2592773/dear-plagiarist-letter-peer-reviewer-who-stole-published-our-manuscript

El Espectador (junio, 2017). China sugiere pena de muerte a investigadores científicos que cometan fraude. El Espectador, Redacción Ciencia. Recuperado de http://www.elespectador.com/ noticias/ciencia/china-sugiere-pena-de-muerte-investigadores-cientificos-que-cometan-fraude-articulo-699975

Ética Psicológica [eticapsicologica.org]. (2017, abril 3). Conflicto de intereses en la publicación científica - Experiencias éticas 04 [Archivo de video]. Recuperado de https://www.youtube. com/watch?v=NQE7mKqL-wo

Ética Psicológica [eticapsicologica.org]. (2017a, abril 3). Plagio y autoplagio en las publicaciones académicas - Experiencias éticas 04 [Archivo de video]. Recuperado de https://www.youtube. com/watch? $=1$ CIErrxvHxM

Ética Psicológica [eticapsicologica.org]. (2017b, abril 6). Manipulación de datos - Experiencias Éticas 04 [Archivo de video]. Recuperado de https:// www.youtube.com/watch? $v=$ RRkHlzTLnBo

Ética Psicológica [eticapsicologica.org]. (2017c, abril 10). Presentación simultánea de artículos académicos - Experiencias Éticas 04 [Archivo de video]. Recuperado de https://www.youtube. $\mathrm{com} /$ watch? $\mathrm{v}=1 \mathrm{CIErrxvHxM}$

Ética Psicológica [eticapsicologica.org]. (2017d, abril 8). Reatractación de artículos en revistas indexadas - Experiencias Éticas 04 [Archivo de video]. Recuperado de https://www.youtube. com/watch? $\mathrm{v}=\mathrm{J}$-yiQTtLf3o

Ética Psicológica [eticapsicologica.org]. (2017e, abril 20). La importancia de la ética para la publicación científica - Experiencias Éticas 04 [Archivo de video]. Recuperado de https:// www.youtube.com/watch? $\mathrm{v}=$ Gpf5V54PIMo\&feature=youtu.be

Falagas, M. E., \& Alexiou, V. G. (2008). The top-ten in journal impact factor manipulation. Archivum Immunologiae et Therapiae Experimenta- lis, 56(4), 223-226. doi:10.1007/s00005-0080024-5

Finelli, C., Crispino, P., Gioia, C., La Sala, N., D’amico, L., La Grotta, M., et al. (2016). The improvement of large High-Density Lipoprotein (HDL) particle levels, and presumably HDL metabolism, depend on effects of low-carbohydrate diet and weight loss. EXCLI Journal, 15, 166-176 (retracted article).

Gómez Ortiz, V., Perilla Toro, L. E., \& Hermosa Rodríguez, A. (2015). Moderación de la Relación Entre Tensión Laboral y Malestar de Profesores Universitarios: Papel del Conflicto y la Facilitación Entre el Trabajo y la Familia. Revista Colombiana de Psicología, 24(1), 185-201. DOI: https://doi.org/10.15446/rcp.v24n1.42081

Gutiérrez, G. A. (2015). Editorial: Fraude académico e institucionalidad. Laberinto, 15(2), 3-6.

Judson, H. F. (2006). Anatomía del fraude científico. Barcelona: Crítica.

Kwok, L. (2005). The White Bull effect: abusive coauthorship and publication parasitism. Journal of Medical Ethics, 31(9), 554-556. doi: 10.1136/jme.2004.010553

Langer, T., Conrad, S., Fishman, L., Gerken, M., Schwarz, S., Weikert, B., et al. (2012). Conflicts of Interest Among Authors of Medical Guidelines: An Analysis of Guidelines Produced by German Specialist Societies. Deutsches Ärzteblatt International, 109(48), 836-842. http:// doi.org/10.3238/arztebl.2012.0836

López López, W. (2014). Editorial: Sobre el plagio, la autoría y otros problemas de ética de las publicaciones. Universitas Psychologica, 13(4), 325-326.

Peterson, S. J., Walumbwa, F. O., Avolio, B. J., \& Hannah, S. T. (2012). RETRACTED: The relationship between authentic leadership and follower job performance: The mediating role of follower positivity in extreme contexts. The Leadership Quarterly, 23(3), 502-516. Retraction notice: https://doi.org/10.1016/j. leaqua.2014.11.001 
Piña, J. A. (2017). Predictores del rendimiento académico en bachilleres, de Gaxiola y colaboradores: ¿un ejemplo de corrupción académica? Enseñanza e Investigación en Psicología, 22(2), 237-256.

RACC (2017). Remoción de artículo. Nota editorial. Revista Argentina de Ciencias del Comportamiento, 9(1). Recuperado de https://revistas. unc.edu.ar/index.php/racc/article/view/16805 Springer (abril, 2017). Retraction Note to multiple articles in Tumor Biology. Recuperado de https://link.springer.com/article/10.1007/ s13277-017-5487-6

Stapel, D. (2014). Faking science: A true story of academic fraud. Disponible en: https://errorstatistics.files.wordpress.com/2014/12/fakingscience-20141214.pdf

Wager, E., Barbour, V. Yentis, S., \& Kleinert, S. (septiembre, 2009). Retraction Guidelines. COPE. Recuperado de https://publicationethics.org/ files/retraction\%20guidelines_0.pdf

Wikipedia (2017). Diederik Stapel. Recuperado de https://en.wikipedia.org/wiki/Diederik_Stapel 
